\section{Weniger Beschneidungen, mehr Infektionen}

In den 1980er-Jahren lag die Beschneidungsrate in den USA bei Neugeborenen noch bei knapp $80 \%$. Seit immer mehr Bundesstaaten dazu übergegangen sind, die Beschneidung nicht mehr über das Medicaid-Programm zu finanzieren derzeit ist das schon in 18 Bundesstaaten der Fall -, ist die Rate auf 55\% im Jahr 2010 gesunken. Das war Anlass für Gesundheitsforscher in Baltimore, in einer Modellrechnung zu überprüfen, wie es sich auf die Infektionsraten und auf die Kosten für das Gesundheitswesen auswirken würde, wenn die Beschneidungsrate auf $10 \%$ sinkt. So niedrig ist sie in den Ländern Westeuropas.

Der Berechnung der Autoren zufolge würden die Gesundheitskosten im Laufe des Lebens eines Mannes um 407 USDollar und einer Frau um 43 US-Dollar steigen. Mehr als drei Viertel der Kosten würden aufgrund der Zunahme von HIVInfektionen bei Männern entstehen. Und: Der Anstieg der für die gesamte Lebensdauer anfallenden direkten Gesundheitsausgaben läge bei mehr als einer halben Milliarde US-Dollar pro Jahr. Jede unterlassene Beschneidung schlägt dabei mit 313 US-Dollar zu Buche.

Die Computerberechnungen ergaben, dass in den USA bei Männern mit einer Erhöhung der Lebenszeitprävalenz von HIV-Infektionen um 12,2\% (fast 4.900 Infizierte) zu rechnen wäre sowie mit einem Anstieg der Lebenszeitprävalenz von Infektionen mit Papillomaviren um 29,1\% (mehr als 57.000 Infizierte).

Auch Frauen wären gefährdet: Die Lebenszeitprävalenz etwa der bakteriellen Vaginose würde um mehr als $50 \%$ steigen, was einer Zunahme von 540.000 Infizierten entspricht. Ähnlich stark wäre die Zunahme der Trichomoniasis-Prävalenz (fast 65.000 Infizierte). Und der Anstieg der Prävalenz von Infektionen mit HPV vom Hochrisikotyp läge bei mehr als 18\% (mehr als 33.000 Infizierte).

Die American Academy of Pediatrics hat jetzt in einem Statement betont, dass die bisherigen Daten für eine generelle Empfehlung der Beschneidung aller männlichen Säuglinge nicht ausreichen [Pediatrics 2012;130: 585-6]. Die Gesellschaft hält es aber für sinnvoll, sie jenen Familien, die eine Beschneidung ihres Säuglings wollen, nicht vorzuenthalten und die Kosten zu erstatten. Peter Leiner

Kacker $S$ et al. Arch Pediatr Adolesc Med 2012; 20: 1-9

\title{
Pityriasis lichenoides: orales Erythromycin als Option
}

Auch im Kindesalter ist Pityriasis lichenoides eine regelmäßig zu beobachtende Hauterkrankung. Gerade hier ist die Verträglichkeit der Therapie von entscheidender Bedeutung.

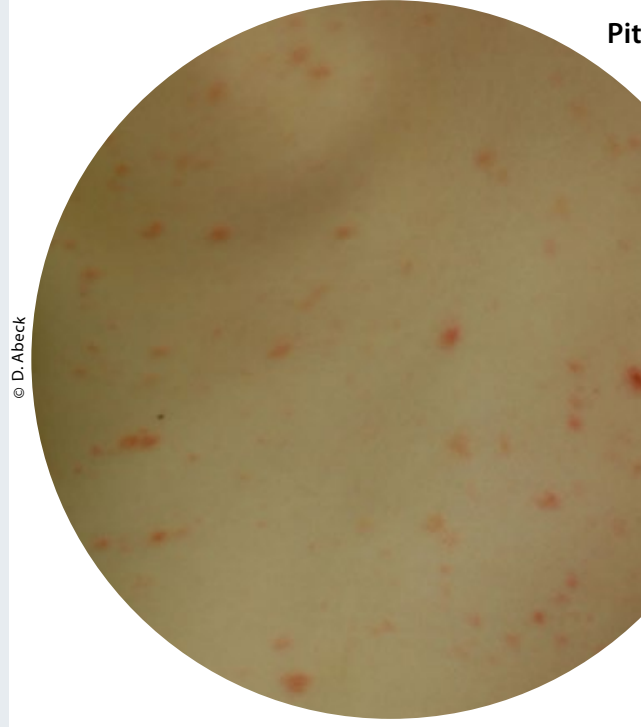

Pityriasis lichenoides: massive Aussaat von entzündlichen Papeln am hinteren Stamm, teilweise mit zentraler Kruste

chung prüften Autoren bei 24 Kindern (15 PLC, 6 PLEVA, 3 PLC/PLEVA) die Wirksamkeit von Erythromycin, wobei eine Tagesdosis von $30-50 \mathrm{mg} / \mathrm{kg}$ auf drei oder vier Einnahmen verteilt wurde. Die Behandlung erstreckte sich über einen Zeitraum von bis zu vier Monaten und erfolgte kontinuierlich bis zum klinischen Ansprechen mit anschließender Dosishalbierung sowie weiterer Gabe über vier Wochen zur Verhinderung eines Rezidivs.

Nach einem Monat zeigte sich ein gu-

D ie Pityriasis lichenoides manifestiert sich in einer akuten (Pityriasis lichenoides et varioliformis acuta, PLEVA) und einer chronischen Variante (Pityriasis lichenoides chronica, PLC). PLEVA ist durch eine Eruption von $\mathrm{Pa}$ peln charakterisiert, die später eine zentrale Hämorrhagie entwickeln und unter Hinterlassung varioliformer Narben abheilen. Bei PLC bestimmen kleine Papeln und Maculae das Bild, die von einer typischen oblatenförmigen Schuppkrause bedeckt sind.

Beim selben Patienten können beide Varianten gleichzeitig bestehen. Derzeit werden antigene Stimuli wie virale Trigger als Ursache diskutiert. Die Erkrankung kann über Jahre bestehen. Subjektive Beschwerden fehlen meist. Die Hautveränderungen sind aber häufig subjektiv belastend, sodass eine Behandlung gewünscht wird. $\mathrm{Zu}$ den in der Literatur beschriebenen Therapien gehören topische Steroide, topische Calcineurininhibitoren (Tacrolimus), orale Antibiotika (Erythromycin und Azithromycin, Tetrazyklin, Cephalosporine), natürliches UV-Licht und orale Immunsuppressiva (Cyclosporin, Methotrexat). In einer retrospektiven Untersuter Therapieerfolg, definiert als Abheilung von mehr als $50 \%$ der Hautveränderungen bei $64 \%$ der Kinder. Nach zwei bzw. drei Monaten stieg dieser Anteil auf 73 bzw. $83 \%$ an. Bei sieben Kindern kam es zur vollständigen Abheilung der Hautveränderungen. Bei drei von 16 auswertbaren Kindern trat ein Rezidiv auf, das signifikant häufiger bei den jüngeren Kindern auftrat (Durchschnittsalter bei zwei Jahren im Vergleich zu acht Jahren für Kinder ohne Rezidiv). Die Behandlung mit Erythromycin wurde in allen Fällen gut vertragen.

Hapa A et al. Childhood pityriasis lichenoides and oral erythromycin. Pediatr Dermatol 2012 May 29. doi: 10.1111/j.1525-1470.2012.01765.x. [Epub ahead of print]

Kommentar: Makrolide verfügen neben der antibiotischen Wirkung auch über immunsuppressive und -modulatorische Eigenschaften. Sie werden, wie auch in dieser Studie gezeigt, sehr gut vertragen. Die orale Erythromycin-Behandlung stellt eine Option im Kindesalter dar, wenn eine Therapie verlangt wird. Sie ist jedoch über mindestens drei Monate durchzuführen bevor eine abschließende Bewertung möglich ist. 